
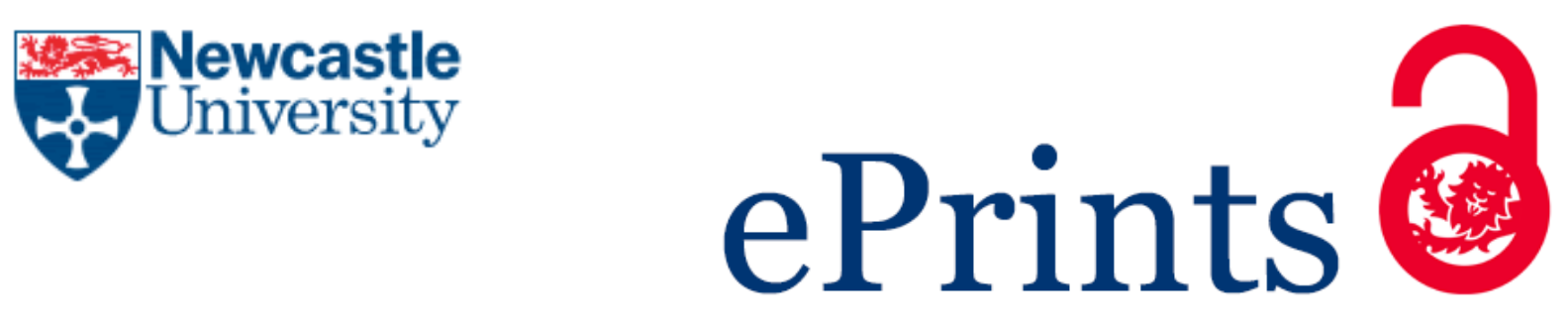

Probert PME, Ebrahimkhani MR, Oakley F, Mann J, Burt AD, Mann DA, Wright MC. A reversible model for periportal fibrosis and a refined alternative to bile duct ligation. Toxicology Research 2014, 3(2), 98-109.

Copyright:

(C) The authors, 2014.

DOI link to article:

http://dx.doi.org/10.1039/C3TX50069A

Date deposited:

$09 / 11 / 2015$

Embargo release date:

28 August 2014

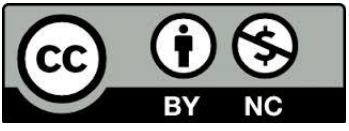

This work is licensed under a Creative Commons Attribution-NonCommercial 3.0 Unported License 


\title{
A reversible model for periportal fibrosis and a refined alternative to bile duct ligation
}

\author{
Philip M. E. Probert, ${ }^{* a}$ Mohammad R. Ebrahimkhani, ${ }^{\text {b }}$ Fiona Oakley, ${ }^{\text {a Jelena Mann, }},{ }^{\text {a }}$ Alastair D. Burt, ${ }^{\text {a }}$ \\ Derek A. Mann, ${ }^{a}$ Matthew C. Wright ${ }^{\text {a }}$ \\ ${ }_{5}$ Received (in $\left.X X X, X X X\right)$ Xth $X X X X X X X X X 20 X X$, Accepted Xth XXXXXXXXX 20XX \\ DOI: 10.1039/b000000x
}

Bile duct ligation (BDL) is commonly employed as a model for hepatic periportal fibrosis. However, $\mathrm{BDL}$ is limited in that it is a severe procedure; is irreversible in practice; the severity of injury cannot be modulated and the procedure is associated with high mortality. Methapyrilene (MP) administration has

10 therefore been investigated as an alternative model. Male rats were subjected to BDL or orally dosed with $150 \mathrm{mg} \mathrm{MP} / \mathrm{kg}$ body weight 3 times per week for up to 6 weeks. Both procedures resulted in increases in serum alkaline phosphatase enzyme levels and periportal liver injury as judged by marked inflammatory cell recruitment to the periportal regions of the liver lobule. Injury in both models was associated with an accumulation of pro-fibrogenic myofibroblast and fibroblast populations - and to ${ }_{15}$ fibrosis - in the periportal regions of the liver lobule. Both procedures also caused an expansion in the number of biliary epithelial cells and a ductular reaction. However, the studies with MP resulted in no rat mortality, in contrast to the BDL procedure. Injury, inflammation, fibrosis and the ductular reaction that occurred in response to MP treatment all reversed within 3 weeks after MP treatment withdrawal. The MP model of periportal fibrosis is therefore a superior - more refined - model of periportal fibrosis than BDL 20 since the degree of injury may be modulated (through alterations in injuring MP dose); the procedure is less severe; is subject to minimal mortality and is reversible (and therefore a more sensitive model to identify effective anti-fibrogenic drugs).

\section{Introduction}

Chronic liver damage caused by a variety of insults (e.g. 25 infections such as hepatitis C; alcohol; obesity; genetic diseases such as Willson's disease; autoimmune diseases such as primary biliary cirrhosis) results in hepatic fibrosis that limits the ability of the liver to regenerate, leading to cirrhosis, hepatocellular carcinoma and end-stage liver failure. ${ }^{1}$ There are currently no 30 treatments for hepatic fibrosis, therefore fibrosis progression is responsible for a significant proportion of the morbidity and mortality associated with liver disease. ${ }^{2}$ Depending on the hepatic insult, the localisation and distribution of hepatic fibrosis may vary within the liver lobule. For example, alcoholic hepatitis 35 produces centrilobular necrosis and fibrosis whereas viral hepatitis and cholestatic liver disease cause hepatic damage and fibrosis emerging from the portal tract (periportal region of the lobule). ${ }^{1}$ Accordingly, in the development of effective antifibrogenic drugs for the liver, there is a need to test for 40 therapeutuic efficacy using at least two experimental models of liver fibrosis - including at least a centrilobular as well as periportal model of fibrosis. 1,3

The most commonly used model for periportal fibrosis is bile 45 duct ligation (BDL), a surgical procedure involving the double ligation of the common bile duct. ${ }^{4}$ The pathology resulting from BDL is similar to that seen in human chronic cholestatic disease:initiation of a ductular reaction and activation of portal tract fibroblasts leading to portal-portal bridging fibrosis. ${ }^{4-6}$ However, ${ }_{50} \mathrm{BDL}$ involves a surgical procedure that requires expertise. Surgery and the severity of the procedure often result in a significant level of mortality. ${ }^{4}$ This high level of mortality limits the long term use of BDL in animals (generally no longer than 4 weeks ). ${ }^{7}$ Additionally, although the procedure can be reversed by 55 additional surgery, this is unlikely to occur due to cost, time and welfare constraints. The procedure in the United Kingdom is therefore classified as "severe" under the Animals (Scientific Procedures) Act 1986.
60 Methapyrilene
(MP)
[N,N-dimethyl-N'-Pyridyl-N'(2- thienylmethyl)-1,2-ethanediamine] is an $\mathrm{H} 1$ receptor antagonist that was used as a sedative and anti-histamine until its withdrawal in the late 1970s, when it was demonstrated to promote hepatocellular carcinoma in chronically-dosed rats. ${ }^{8}$ Subsequent 65 work demonstrated that MP is a periportal hepatotoxin in rats. ${ }^{9-17}$ MP is bio-activated in the liver by cytochromes P450, leading to the production of a reactive intermediate (potentially a thioether of MP S-oxide). ${ }^{14,18}$ The intermediate(s) is thought to disrupt mitochondria leading to hepatocyte apoptosis and necrosis and 70 consequently hepatic inflammation. ${ }^{14,16,19}$ 
Based on the observation that MP causes periportal hepatic damage, we hypothesised that the chronic dosing of MP to rats would lead to a less severe, more controllable and reversible 5 periportal fibrosis. We therefore compared the fibrosis generated by BDL and MP in rats. The data in this paper demonstrate for the first time that MP produces a qualitatively similar fibrosis to BDL. MP fibrosis therefore represents a more refined and superior model of hepatic periportal fibrosis than the BDL model.

10

\section{Results}

\section{MP treatment caused a cholestatic injury and an increase in 15 liver weight.}

To determine the effects of chronic MP dosing in rats, MP was administered by oral gavage at a dose of $150 \mathrm{mg} \mathrm{MP} / \mathrm{kg}$ body weight 3 times per week. This dose was chosen because it has 20 previously been shown to cause moderate hepatic damage after acute administration. $^{20}$ Table 1 shows that this dose of MP did not cause a loss of body weight, but similarly to BDL, caused a significant decrease in gain of weight compared to their respective controls. Figure 1a indicates that MP treatment 25 significantly increased serum ALP levels with non-significant increases in ALT and AST serum enzyme levels. In contrast, BDL significantly increased serum ALP, ALT and AST levels compared to sham treated rats. Serum $\gamma$-glutamyl transpeptidase (gGT) was undetectable in control animals (vehicle and sham 30 controls) but was detectable in 3 of 5 MP treated rats and all rats subject to 14 days BDL (data not shown). Measurement of liver weight indicated that MP administration and BDL were also associated with a significant increase in liver weight as a proportion of total body weight (Figure 1b). There was no 35 mortality associated with the administration of MP, whereas BDL caused $30 \%$ mortality after 14 days.

\section{MP treatment caused portal tract inflammation and initiated an hepatic regenerative response.}

40

To determine whether liver injury caused by MP administration resulted in qualitatively similar pathological lesions, MP was administered to rats for 3 or 6 weeks, with an additional group allowed to recover after 3 weeks MP treatment to determine 45 whether pathological changes were reversible.

Liver sections from MP treated and control animals were stained with H\&E and compared to liver sections from rats culled 14 days after BDL surgery. Control animals showed occasional 50 infiltrating leucocytes in the periportal region whereas both MP and BDL treated rats showed cell injury and necrosis in the periportal regions accompanied with marked significant increases in periportal inflammatory cells (Figure 2). MP treatment for 6 weeks did not significantly increase inflammatory cell 55 recruitment over that present at 3 weeks. However, staining sections for neutrophils indicates that there was a progressive increased recruitment of neutrophils to the periportal regions of the liver lobule (Supplementary Figure 1). These adverse effects to MP were specific to the liver as judged macroscopically (data 60 not shown). Limited pathological examination of non-hepatic tissues including kidney (Figure 2), lung and spleen (Supplementary Figure 2) after 6 weeks of MP treatment support the suggestion that the liver is a target organ for MP toxicity.

${ }_{65}$ Expression of the marker for proliferating cells - PCNA $^{21}-$ indicates that both BDL and MP administration resulted in an increase in PCNA positive hepatocytes and cholangiocytes, suggesting these cells undergo mitosis in response to injury (Figure 3, see also Supplementary Figure 3). Figure 3 also shows 70 that this proliferative response reversed in MP treated rats within 3 weeks post withdrawal of MP.

\section{MP treatment caused an expansion of pro-fibrogenic cells and periportal fibrosis.}

Myofibroblasts and fibroblasts are recognised to play a fundamental role in deposition of extracellular matrix during hepatic fibrogenesis. ${ }^{22-24}$ Of these populations, portal tract fibroblasts have been implicated as the primary fibrogenic cell 80 population involved in deposition of fibrotic extracellular matrix during the initial stages of periportal fibrosis. ${ }^{25}$ Portal tract fibroblasts have been shown to express vimentin. ${ }^{26}$

Figure 4 demonstrates that a low number of cells positive for 85 vimentin were present in liver sections from control animals and that these cells were evenly spread throughout the hepatic lobule. After MP treatment or BDL, the number of vimentin positive cells was significantly increased around periportal regions. Significantly more periportal vimentin staining was seen after 6 90 weeks MP than after 3 weeks MP. The significant increase in the number of vimentin positive cells after 3 weeks MP treatment was reversed after a 3 week recovery period (Figure 4).

Myofibroblasts are a pro-fibrogenic cell population associated 95 with peri-sinusoidal and centrilobular injury,,$^{22,23,27}$ and express the marker $\alpha$-SMA. ${ }^{28}$ Figure 5 demonstrates that only smooth muscle cells surrounding portal arterioles expressed $\alpha$-SMA in liver sections from control rats. In BDL and MP treated rats, there was a significant increase in the number of periportal $\alpha$-SMA 100 positive cells. Liver sections from rats treated with MP for 6 weeks had significantly more periportal $\alpha$-SMA positive cells than rats treated for 3 weeks. Similar to periportal vimentin staining, the significant increase in the number of periportal $\alpha$ SMA positive cells seen in rats after 3 weeks MP was reversible 105 after 3 weeks vehicle treatment.

Sirius red staining for collagen fibres showed that both BDL and MP treatment induced significant periportal fibrosis (Figure 6). Since biliary fibrosis severity can be somewhat heterogeneous 110 throughout the liver, image analysis was performed (by an experimenter blinded to the identity of the treatments as outlined in the methods section) on 10 randomly selected portal tracts per section per rat to robustly test the effects of MP on fibrogenesis and recovery. Sirius red staining quantification (Figure 6) and 115 hepatic hydroxyproline contents (Supplementary Figure 4) confirm MP treatment for both 3 and 6 weeks caused a significant 
increase in hepatic collagen content and therefore fibrosis. Prolonged treatment with MP (6 weeks) led to a significant increase in periportal fibrosis compared to that seen after 3 weeks and 14 days BDL (Figure 6). The increase in periportal fibrosis 5 after 3 weeks was reversible as rats treated with 3 weeks MP followed by 3 weeks vehicle no longer showed significant periportal collagen deposition. Central veins appeared similar between MP treated and control animals indicating that MP primarily caused periportal damage and fibrosis.

10

\section{MP treatment caused a ductular reaction.}

Ductular reaction is a regenerative response involving the significant expansion of CK-19 positive cholangiocytes and it is 15 thought to play an important role in fibrogenesis. ${ }^{29}$ Figure 7 shows that both BDL and MP gave rise to a ductular reaction with clear evidence of bile duct expansion. The reaction was significantly more severe after 6 weeks than 3 weeks of MP treatment, with a reverse in ductular reaction within 3 weeks after ${ }_{20}$ MP withdrawal (Figure 7).

Chronic MP treatment caused a significant and reversible upregulation of expression of genes associated with fibrogenesis.

${ }_{25}$ The expression of genes associated with fibrogenesis was measured to place the changes observed in the liver after MP treatment in a more quantitative context to BDL. Table 2 demonstrates that both BDL and MP treatment up-regulated the expression of a variety of mRNAs associated with fibrogenesis $30 \alpha$-SMA, collagen 1A1 (COL1A1), MMP-2, TGF- $\beta 1$, TGF- $\beta 2$, TIMP-1 and vimentin. The expression of these genes also returned to basal levels in the group of rats treated with MP for 3 weeks followed by a 3 week recovery.

\section{Discussion}

A major complicating factor in chronic liver disease is fibrosis, for which there are no treatments indicated except 40 transplantation. Fibrosis therefore remains a major determining factor in the high rate of mortality arising from chronic liver disease worldwide. ${ }^{1,2}$ Models of periportal fibrosis are important in research into anti-fibrogenics. This is because it is recommended that anti-fibrogenics are tested in both at least one

45 centrilobular and periportal model of fibrosis (due to the different cell types contributing to fibrogenesis ${ }^{1,3,25}$ ). In view of the limitations associated with the BDL model, chronic MP dosing was investigated as an alternative.

${ }_{50}$ Chronic MP administration of rats significantly elevated serum ALP levels suggesting that MP caused a cholestatic injury with evidence of a trend toward hepatocyte necrosis (Figure 1). BDL conversely significantly elevated all liver serum markers. The absence of significant ALT and AST serum activity after MP 55 treatment is likely because the dose chosen caused less severe injury compared to BDL and hepatic regenerative pathways were sufficient to remove and replace damaged hepatocytes between MP doses. Importantly, MP treatment unlike BDL caused no mortality and there were no complications associated with the 60 procedure.

Qualitative similarities between the pathology of BDL- and MPinduced fibrosis were seen at several levels. Inflammation is generally a pre-requisite for the development of fibrosis. ${ }^{1}$ 65 Analysis of liver histology by H\&E staining (Figure 2) showed that MP and BDL caused significant increases in the number of inflammatory cells in and around the portal tracts, which has been seen previously after both MP and BDL treatment. ${ }^{9,10,30}$ PCNA immunostaining showed that 6 weeks MP treatment and BDL 70 both significantly increased hepatocyte and cholangiocyte proliferation (Figure 3) demonstrating that both treatments led to hepatic damage and activation of hepatic regenerative pathways, although any role for the bi-potential progenitor (oval) cell, if any, in this process could not be determined from the studies as 75 designed.

In terms of fibrogenic cells, the numbers of myofibroblasts and portal tract fibroblasts in the periportal region positively correlated with the length of MP administration and their 80 numbers significantly decreased following the cessation of MP treatment (Figures 4 and 5). Sirius red staining of liver sections from treated rats demonstrated that MP administration was associated with significant increased expression of COL1A1 and periportal deposition of collagen (Table 2 and Figure 6) in a 85 manner that mirrored the changes in fibrogenic cell populations. Based on sirius red stained liver sections, the severity of fibrosis in BDL sits between the severities seen between 3 and 6 weeks of MP treatment.

90 Spontaneous reversal of fibrosis is recognised to occur in some patients where the underlying cause of hepatic insult is successfully treated, however the processes that underlie this reversal are poorly understood. ${ }^{1}$ The severity of periportal fibrosis in MP treated rats correlated with the number of pro95 fibrogenic cells. The resolution of fibrosis seen in rats treated with MP for 3 weeks followed by vehicle for 3 weeks is therefore likely to be the result of apoptosis or reversal of the profibrogenic phenotype of these populations. This may result from a decline in inflammation and associated pro-fibrogenic growth 100 factors released from inflammatory cells. Expression of hepatic TGF- $\beta 1$ and TIMP- 1 mRNAs to control levels in rats treated with MP for 3 weeks followed by vehicle for 3 weeks (Table 2), supports this hypothesis. The ease of reversal in this model facilitates its use as a method to study the reversal of periportal 105 fibrosis, along with other rodent models of xenobiotic induced biliary fibrosis, ${ }^{31,32}$ as an alternative to BDL reversal.

Ductular reaction promotes fibrosis development as ductules are pro-fibrogenic through the release of a range of chemokines, 110 cytokines and growth factors that promote inflammation and fibrosis..$^{29,33}$ The expansion of ductal cells was quantified by CK19 staining and showed that MP caused a ductular reaction similarly to BDL in rats (Figure 7). The ductular reaction induced by MP was also shown to be reversible. Chronic MP treatment 115 caused liver damage and activation of a ductular reaction in a more gradual manner relative to BDL. The use of a MP model for 
the study of this phenomenon would therefore allow for a longer time frame for study of the early stages of a ductular reaction.

It is clear from much of the data in this paper that BDL resulted 5 in higher levels of many biomarkers of liver disease when compared to MP treatment at the dose employed. This is because BDL causes severe, constant and uncontrollable hepatic damage which likely is the cause for the high rate of mortality associated with the procedure. Dosing of MP is a more controllable model 10 which also allows for some degree of hepatic regeneration to occur. There is also the option of modifying the doses employed to increase the severity of injury. The MP model may therefore be a superior model of human hepatic periportal fibrosis. This is primarily because in humans with chronic hepatic disease, 15 fibrosis develops gradually and is frequently reversible when the underlying cause is removed. ${ }^{3}$

In terms of potential drug interactions, the dosing of MP has been shown to cause a reduction in CYP2C11 and CYP3A activity in 20 rats in vivo although the activity of the CYP1A and CYP2B families are unaffected by MP treatment. ${ }^{19}$ Knockout of the H1 receptor has been shown to have no effect on hepatic injury in a murine model of liver injury ${ }^{34}$ - the antagonistic action of MP on the $\mathrm{H} 1$ receptor is therefore not predicted to have a significant 25 effect on MP induced hepatic damage. If the efficacy of antifibrogenics is being tested in periportal fibrosis generated by MP, drug interactions should be considered in analysis of results. MP is an $\mathrm{H} 1$ antagonist in the rat ${ }^{35}$ and mast cells increase in number in fibrotic tissue $\mathrm{e}^{36}$ and contribute to the progression of fibrosis via 30 the release of mediators such as histamine, tryptase, heparin and fibroblast growth factors. ${ }^{37}$ Furthermore, the proliferation of small cholangiocytes has been shown to be promoted by histamines. ${ }^{38}$ Most compounds used in fibrosis studies will have "off target" effects with respect to toxicity that may also 35 modulate subsequent fibrogenic responses. With drugs, there may be effects associated with known pharmacological targets (e.g. paracetamol and cyclooxygenase inhibition ${ }^{39}$ ). However, even the commonly employed centrilobular hepatotoxin $\mathrm{CCl}_{4}$ (which injures hepatocytes via a CYP2E1-mediated lipid 40 peroxidation mechanism ${ }^{1}$ ) has been shown to inhibit protein kinase $\mathrm{C}$ activity by $50 \%$ in cells at a concentration of $1 \mathrm{uM}{ }^{40}$

It has been shown here for the first time that chronic administration of MP leads to development of reversible hepatic 45 periportal fibrosis with characteristics qualitatively similar to that seen after BDL in male rats, but without the requirement for surgery, distress and high mortality rates. Use of MP as an alternative to BDL would allow for modulation of fibrosis severity through changing the dosing regimen, whilst allowing 50 fibrosis formation to be reversed if required. Chronic MP administration is therefore a refined alternative to BDL producing a reproducible model of reversible periportal fibrosis in rats.

\section{${ }_{55}$ Materials and methods}

\section{Animal studies}

Male Sprague-Dawley rats $(230-250 \mathrm{~g}$ body weight) were purchased from Charles River (Kent, UK) and housed in 60 accordance with Home Office guidelines. Rats were kept no more than 5 per cage. Food and water for animals were available ad libitum and conditions were kept at $23^{\circ} \mathrm{C} \pm 1^{\circ} \mathrm{C}$ on a 12 hour light/12 hour dark cycle at $47 \%$ humidity. Rats were dosed with $150 \mathrm{mg}$ methapyrilene hydrochloride (Sigma, Poole, UK)/kg body 65 weight from a $150 \mathrm{mg} / \mathrm{ml}$ phosphate buffer saline (PBS, Sigma) stock by oral gavage 3 times per week. For the initial pilot study, rats were dosed with MP or control vehicle $(1 \mathrm{ml} / \mathrm{kg}$ body weight PBS) for 3 weeks. For the reversibility study, rats were dosed for 3 weeks (with 3 weeks for recovery) or continuously for 6 weeks. 70 Body weight was measured 3 times per week. After 0,21 or 42 days, the rats were killed by $\mathrm{CO}_{2}$ asphyxiation followed by cervical dislocation. Tissues and bloods were then removed for subsequent analysis. Bile duct ligation was carried out by double ligation of the common bile duct essentially as previously 75 described on Sprague-Dawley rats. ${ }^{40,41}$ Sham operated rats underwent the same procedure, but the bile duct was not ligated. After surgery rats were kept in a warm room and closely monitored. BDL rats were killed and livers harvested at 14 days post surgery. Experiments were performed under a UK Home ${ }_{80}$ Office licence with Local Ethics Committee approval.

\section{Serum biochemistry}

Serum was prepared from blood samples by centrifugation and measured for serum (alanine aminotransferase (ALT), alkaline 85 phosphatase (ALP) and aspirate aminotransferase (AST) essentially as previously described. ${ }^{42}$

\section{Histology and immunohistochemistry}

Organs were fixed in $10 \%$ buffered formalin and processed as 90 previously described. ${ }^{42}$ Haematoxylin and eosin $(\mathrm{H} \& \mathrm{E})$ and sirius red staining were carried out as previously described. ${ }^{42}$ Neutrophils were identified using a naphthol AS-D chloroacetate esterase stain (Sigma Chemical Company, Poole, UK) following the recommended protocol. Immunohistochemistry for 95 proliferating cell nuclear antibody (mouse anti-PCNA, Abcam, Cambridge, UK) $\alpha$-smooth muscle actin ( $\alpha$-SMA, rabbit anti- $\alpha$ SMA, Abcam), cytokeratin-19 (CK-19, rabbit anti-CK-19, Abcam) and vimentin (rabbit anti-vimentin, Abcam) was performed as previously described. ${ }^{42}$ For all immunostaining, 100 sodium citrate antigen retrieval was used to expose epitopes and primary antibodies were used at a 1:250 dilution. Di-amino benzidine (Dako, Ely, UK) was used to visualise secondary antibody binding to primary antibodies (anti-mouse/HRP or anti rabbit/HRP, 1:250 dilution, Dako). All sections were 105 counterstained with haematoxylin prior to mounting.

Inflammatory cell or neutrophil infiltration were calculated by counting the number of periportal leucocytes in and around 6 random portal tracts per section from the H\&E or esterase stained 110 sections respectively. PCNA immunostaining was quantified by counting the number of PCNA positive hepatocytes and cholangiocytes around 5 random portal tracts per section at $\mathrm{x} 20$ magnification. Sirius red, $\alpha$-SMA, CK-19 and vimentin staining were quantified by densitometry analysis using Qwin software 115 (Leica, Wetzlar, Germany) after manually specifying the threshold for positive staining. The threshold was selected to 
ensure consistent measurement of staining across different sections. Analysis was carried out at x10 magnification, focusing on portal tracts in 10 random fields per section. All quantifications were carried out by a researcher blinded to the 5 treatment groups. For whole liver lobule sirius red quantification, slides were scanned using a Leica SCN400 slide scanner and then analysed for positive staining using the accompanying slidepath software.

\section{${ }_{10}$ Quantitative real-time PCR}

RNA was purified from liver samples using TRIzol (Life Technologies, Paisley, UK), DNAse-treated using RQ1 DNAse (Promega, Southampton, UK) and complementary DNA then synthesised using MML-V reverse transcriptase (Promega), 15 following the manufacturer's instructions. Quantitative real-time PCR was carried out using SYBR green (Sigma) on a 7500 Fast thermocycler (Applied biosciences, Paisley, UK) The sequences of the primers used were: rCOL1A1, US 5'TGTGTTGCTGAAAGACTACCTCGT -3' DS 5'20 AGTTGCCCCGGTGACACACAA -3'; rTGF- $\beta 1$, US 5'CGAGCCCGAGGCGGACTACT -3' DS 5'ATAGATTGCGTTGTTGCGGTCCACC -3'; rTGF- $\beta 2$, US 5'TTCAGAATCGTCCGCTTCGAT -3 ' DS 5'TTGTTCAGCCACTCTGGCCTT -3'; rMMP2, 5'${ }_{25}$ GCACCGTCGCCCATCATCAAGT -3 ' DS 5'TTGCGGGGAAAGAAGTTGTAGT -3'; rTIMP1, US 5'GACCACCTTATACCAGCGTT -3 , DS 5'GTCACTCTCCAGTTTGCAAG -3'; rVimentin, US 5'CAGGCCACCTCGTCCTTCGAAG -3' DS 5'30 TGTGCCGGAGCCACCGAACAT -3'; r $\alpha-S M A$, US 5'TGCCATGTATGTGGCTATTCA $-3^{\prime}$ DS 5'ACCAGTTGTACGTCCAGAAGC -3'. Relative expression was calculated based on the $\Delta \Delta \mathrm{Ct}$ method with gene expression normalised to $18 \mathrm{~S}$ rRNA expression.

35

\section{Hydroxyproline assay}

Hydroxyproline assays were carried out using the Quickzyme hydroxyproline assay kit (2B Scientific, Upper Heyford, UK). Samples were prepared by incubating liver samples in $6 \mathrm{M} \mathrm{HCl}$ $40(10 \mathrm{mg}$ wet weight/100ul $\mathrm{HCl})$ overnight at $110^{\circ} \mathrm{C}$. Following acid hydrolysis, the samples were centrifuged at 13,000rpm for 15 minutes in a desktop centrifuge and the supernatant retained for the assay.

\section{${ }_{45}$ Statistics}

Two-tailed Student's T-tests were used to test for statistical significance between groups. Significance was achieved where $p$ $<0.05$.

\section{${ }_{50}$ Acknowledgements}

This work was supported by grants from the National Centre for the Refinement, Reduction and Replacement of Animals in Research (NC3Rs) and The Wellcome Trust (grant number ${ }_{55}$ WT086755MA to M.C.W, A.D.B and D.A.M, and grant WT084961MA to F.O, D.A.M and M.C.W). P.M.E.P is supported by a studentship award from the NC3Rs.

\section{References}

60

1. K. Wallace, A. D. Burt and M. C. Wright, Liver fibrosis. Biochem J, 2008, 411, 1-18.

2. Y. S. Lim and W. R. Kim, The global impact of hepatic fibrosis and end-stage liver disease. Clin Liver Dis, 2008, 12, 733-746, vii.

3. V. Hernandez-Gea and S. L. Friedman, Pathogenesis of liver fibrosis. Annu Rev Pathol, 2011, 6, 425-456.

4. A. Symeonidis and E. G. Trams, Morphologic and functional changes in the livers of rats after ligation or excision of the 70 common bile duct. The American journal of pathology, 1957, 33, 13-27.

5. B. Tuchweber, A. Desmouliere, M. L. Bochaton-Piallat, L. Rubbia-Brandt and G. Gabbiani, Proliferation and phenotypic modulation of portal fibroblasts in the early stages of

75 cholestatic fibrosis in the rat. Lab Invest, 1996, 74, 265-278.

6. O. Tarcin, M. Basaranoglu, V. Tahan, G. Tahan, I. Sucullu, N. Yilmaz, G. Sood, N. Snyder, G. Hilman, C. Celikel and N. Tozun, Time course of collagen peak in bile duct-ligated rats. BMC Gastroenterol, 2011, 11, 45.

80 7. P. Georgiev, W. Jochum, S. Heinrich, J. H. Jang, A. Nocito, F. Dahm and P. A. Clavien, Characterization of time-related changes after experimental bile duct ligation. Br J Surg, 2008, 95, 646-656.

8. W. Lijinsky, M. D. Reuber and B. N. Blackwell, Liver tumors 85 induced in rats by oral administration of the antihistaminic methapyrilene hydrochloride. Science, 1980, 209, 817-819.

9. L. M. Brennan and D. A. Creasia, The effects of methapyrilene hydrochloride on hepatocarcinogenicity and pentobarbital-induced sleeping time in rats and mice. Toxicol Appl Pharmacol, 1982, 66, 252-258.

10. M. E. Graichen, D. A. Neptun, J. G. Dent, J. A. Popp and T. B. Leonard, Effects of methapyrilene on rat hepatic xenobiotic metabolizing enzymes and liver morphology. Fundam Appl Toxicol, 1985, 5, 165-174.

95 11. K. L. Steinmetz, C. K. Tyson, E. F. Meierhenry, J. W. Spalding and J. C. Mirsalis, Examination of genotoxicity, toxicity and morphologic alterations in hepatocytes following in vivo or in vitro exposure to methapyrilene. Carcinogenesis, 1988, 9, 959-963.

100 12. F. C. Richardson, D. M. Copple and P. I. Eacho, Effects of methapyrilene on DNA synthesis in mice and rats following continuous dietary exposure. Carcinogenesis, 1992, 13, 2453 2457.

13. M. L. Cunningham, L. L. Pippin, N. L. Anderson and M. L. Wenk, The hepatocarcinogen methapyrilene but not the analog pyrilamine induces sustained hepatocellular replication and protein alterations in F344 rats in a 13-week feed study. Toxicology and applied pharmacology, 1995, 131, 216-223.

110 14. G. S. Ratra, S. Cottrell and C. J. Powell, Effects of induction and inhibition of cytochromes P450 on the hepatotoxicity of methapyrilene. Toxicol Sci, 1998, 46, 185-196.

15. H. K. Hamadeh, B. L. Knight, A. C. Haugen, S. Sieber, R. P. Amin, P. R. Bushel, R. Stoll, K. Blanchard, S. Jayadev, R. W.

115 Tennant, M. L. Cunningham, C. A. Afshari and R. S. Paules, Methapyrilene toxicity: anchorage of pathologic observations 
to gene expression alterations. Toxicol Pathol, 2002, 30, 470482.

16. A. E. Mercer, S. L. Regan, C. M. Hirst, E. E. Graham, D. J. Antoine, C. A. Benson, D. P. Williams, J. Foster, J. G. Kenna and B. K. Park, Functional and toxicological consequences of metabolic bioactivation of methapyrilene via thiophene Soxidation: Induction of cell defence, apoptosis and hepatic necrosis. Toxicol Appl Pharmacol, 2009, 239, 297-305.

17. C. C. Priestley, S. Regan, B. Kevin Park and D. P. Williams,

10 The genotoxic potential of methapyrilene using the alkaline Comet assay in vitro and in vivo. Toxicology, 2011, 290, 249-257.

18. E. E. Graham, R. J. Walsh, C. M. Hirst, J. L. Maggs, S. Martin, M. J. Wild, I. D. Wilson, J. R. Harding, J. G. Kenna, R. M. Peter, D. P. Williams and B. K. Park, Identification of the thiophene ring of methapyrilene as a novel bioactivationdependent hepatic toxicophore. J Pharmacol Exp Ther, 2008, 326, 657-671.

19. G. S. Ratra, W. A. Morgan, J. Mullervy, C. J. Powell and M.

20 C. Wright, Methapyrilene hepatotoxicity is associated with oxidative stress, mitochondrial disfunction and is prevented by the Ca2+ channel blocker verapamil. Toxicology, 1998, 130, 79-93.

20. G. S. Ratra, C. J. Powell, B. K. Park, J. L. Maggs and S.

25 Cottrell, Methapyrilene hepatotoxicity is associated with increased hepatic glutathione, the formation of glucuronide conjugates, and enterohepatic recirculation. Chem Biol Interact, 2000, 129, 279-295.

21. P. A. Hall, D. A. Levison, A. L. Woods, C. C. Yu, D. B. 30 Kellock, J. A. Watkins, D. M. Barnes, C. E. Gillett, R. Camplejohn, R. Dover and et al., Proliferating cell nuclear antigen (PCNA) immunolocalization in paraffin sections: an index of cell proliferation with evidence of deregulated expression in some neoplasms. The Journal of pathology, 35 1990, 162, 285-294.

22. J. P. Iredale, Models of liver fibrosis: exploring the dynamic nature of inflammation and repair in a solid organ. J Clin Invest, 2007, 117, 539-548.

23. S. L. Friedman, Hepatic stellate cells: protean,

40 multifunctional, and enigmatic cells of the liver. Physiol Rev, 2008, 88, 125-172.

24. S. T. Magness, R. Bataller, L. Yang and D. A. Brenner, A dual reporter gene transgenic mouse demonstrates heterogeneity in hepatic fibrogenic cell populations.

45 Hepatology, 2004, 40, 1151-1159.

25. G. Ramadori and B. Saile, Portal tract fibrogenesis in the liver. Laboratory investigation; a journal of technical methods and pathology, 2004, 84, 153-159.

26. L. Libbrecht, D. Cassiman, V. Desmet and T. Roskams, The

50 correlation between portal myofibroblasts and development of intrahepatic bile ducts and arterial branches in human liver. Liver, 2002, 22, 252-258.

27. S. L. Friedman, F. J. Roll, J. Boyles and D. M. Bissell, Hepatic lipocytes: the principal collagen-producing cells of

55 normal rat liver. Proc Natl Acad Sci U S A, 1985, 82, 86818685 .

28. T. Nouchi, Y. Tanaka, T. Tsukada, C. Sato and F. Marumo, Appearance of alpha-smooth-muscle-actin-positive cells in hepatic fibrosis. Liver, 1991, 11, 100-105.

60 29. S. S. Glaser, E. Gaudio, T. Miller, D. Alvaro and G. Alpini, Cholangiocyte proliferation and liver fibrosis. Expert Rev Mol Med, 2009, 11, e7.

30. J. M. Saito and J. J. Maher, Bile duct ligation in rats induces biliary expression of cytokine-induced neutrophil

65 chemoattractant. Gastroenterology, 2000, 118, 1157-1168.

31. P. Fickert, U. Stoger, A. Fuchsbichler, T. Moustafa, H. U. Marschall, A. H. Weiglein, O. Tsybrovskyy, H. Jaeschke, K. Zatloukal, H. Denk and M. Trauner, A new xenobioticinduced mouse model of sclerosing cholangitis and biliary fibrosis. Am J Pathol, 2007, 171, 525-536.

32. H. M. Golbar, T. Izawa, C. Ichikawa, M. Tanaka, V. Juniantito, O. Sawamoto, M. Kuwamura and J. Yamate, Slowly progressive cholangiofibrosis induced in rats by alpha-naphthylisothiocyanate (ANIT), with particular references to characteristics of macrophages and myofibroblasts. Experimental and toxicologic pathology : official journal of the Gesellschaft fur Toxikologische Pathologie, 2013.

33. A. S. Gouw, A. D. Clouston and N. D. Theise, Ductular 80 reactions in human liver: diversity at the interface. Hepatology, 2011, 54, 1853-1863.

34. T. Masaki, S. Chiba, H. Tatsukawa, H. Noguchi, T. Kakuma, M. Endo, M. Seike, T. Watanabe and H. Yoshimatsu, The role of histamine $\mathrm{H} 1$ receptor and $\mathrm{H} 2$ receptor in LPSinduced liver injury. FASEB J, 2005, 19, 1245-1252.

35. S. J. Hill and J. M. Young. Histamine H1-receptors in the brain of the guinea-pig and the rat: differences in ligand binding properties and regional distribution. Br J Pharmacol, 1980, 68, 687-96.

90 36. D.J. Farrell, J.E. Hines, A.F. Walls et al. Intrahepatic mast cells in chronic liver diseases. Hepatology, 1995, 22, 11751181.

37. M. Yamashiro, W. Kouda, N. Kono et al. Distribution of intrahepatic mast cells in various hepatobiliary disorders. An 95 immunohistochemical study. Virchows Arch, 1998, 433, 471-479.

38. H. Francis, S. Glaser, S. Demorrow, E. Gaudio, Y. Ueno, J. Venter, D. Dostal, P, Onori, A. Franchitto, M. Marzioni, S. Vaculin, B. Vaculin, K. Katki, M. Stutes, J. Savage and G. Alpini. Small mouse cholangiocytes proliferate in response to H1 histamine receptor stimulation by activation of the IP3/CaMK I/CREB pathway. Am J Physiol Cell Physiol, 2008, 295, C499-513.

39. M. Ouellet and M. D. Percival. Mechanism of acetaminophen 105 inhibition of cyclooxygenase isoforms. Arch Biochem Biophys, 2001, 387, 273-80.

40. S. N. Pentyala, P. J. Vig, B.S. Sekhon and D. Desaiah. Effect of carbon tetrachloride on inositol 1,4,5-trisphosphate dependent and independent regulation of rat brain microsomal Ca2+ flux. Cell Signal, 1994, 6, 561-7.

40. M. R. Ebrahimkhani, S. Kiani, F. Oakley, T. Kendall, A. Shariftabrizi, S. M. Tavangar, L. Moezi, S. Payabvash, A. Karoon, H. Hoseininik, D. A. Mann, K. P. Moore, A. R. Mani and A. R. Dehpour, Naltrexone, an opioid receptor antagonist, attenuates liver fibrosis in bile duct ligated rats. Gut, 2006, 55, 1606-1616. 
41. M. R. Ebrahimkhani, F. Oakley, L. B. Murphy, J. Mann, A. Moles, M. J. Perugorria, E. Ellis, A. F. Lakey, A. D. Burt, A. Douglass, M. C. Wright, S. A. White, F. Jaffre, L. Maroteaux and D. A. Mann, Stimulating healthy tissue regeneration by 5 targeting the 5-HT(2)B receptor in chronic liver disease. Nature medicine, 2011, 17, 1668-1673.

42. C. J. Marek, S. J. Tucker, D. K. Konstantinou, L. J. Elrick, D. Haefner, C. Sigalas, G. I. Murray, B. Goodwin and M. C. Wright, Pregnenolone-16alpha-carbonitrile inhibits rodent

10 liver fibrogenesis via PXR (pregnane $\mathrm{X}$ receptor)-dependent and PXR-independent mechanisms. Biochem J, 2005, 387, 601-608.

\section{Inserting Graphics}

15 Use the buttons on the graphics dialog (accessed from the main toolbar by pressing the "Insert Graphics" button) to insert the required template text. It is recommended that graphics are inserted once the text is completed, as the text after graphic insertion will cause the graphics to move unpredicatably.

${ }_{20}$ Graphics should be inserted at the top of the page where they are first mentioned (unless they are equations, which appear in the flow of the text). Place the cursor at the beginning of the first line in either of the columns and press the required button.

\section{Conclusions}

25 The conclusions section should come at the end of article.

\section{Notes and references}

${ }^{a}$ Institute of Cellular Medicine, Newcastle University, Newcastle Upon Tyne,UK. Tel: E-mail:M.C.Wright@ncl.ac.uk

$30^{b}$ Department of Biochemistry, Smilow Research Center, New York University School of Medicine, New York, New York 10016, USA. ${ }^{*}$ Corresponding author: Institute of Cellular Medicine, Level 4 Leech Building, Medical School, Newcastle University, Framlington Place, Newcastle Upon Tyne, UK NE2 4HH. Tel (44) 191222 7745; Fax (+44) 35191222 7179; Email p.m.e.probert@ncl.ac.uk

$\dagger$ Electronic Supplementary Information (ESI) available: [details of any supplementary information available should be included here]. See DOI: $10.1039 / b 000000 x /$

40 
Table 1: Total body weight changes in rats treated with MP or subjected to BDL.

\begin{tabular}{lc}
\hline Group & $\begin{array}{c}\text { \%age body weight change over } 2 \\
\text { weeks (BDL) or 3 weeks (MP) }\end{array}$ \\
\hline Vehicle & $112.4 \pm 3.42$ \\
MP & $102.7 \pm 2.97^{*}$ \\
Sham & $113.2 \pm 11.9$ \\
BDL & $102.2 \pm 3.73^{*}$ \\
\hline
\end{tabular}

5 Values are mean and SD, *significantly different compared to respective controls at $\mathrm{p}<0.05$. For vehicle, $\mathrm{n}=3$; for $\mathrm{MP}$ and sham, $\mathrm{n}=5$; for BDL, $\mathrm{n}=8$. 
Table 2. Whole liver changes in gene expression after MP treatment or 14 day BDL as measured by qPCR.

\begin{tabular}{|c|c|c|c|c|c|c|c|}
\hline \multirow[t]{2}{*}{ GROUP } & \multicolumn{7}{|c|}{ mRNA transcript (fold control levels) } \\
\hline & $\alpha$-SMA & COL1A1 & MMP-2 & TGF- $\beta 1$ & TGF- $\beta 2$ & TIMP-1 & Vimentin \\
\hline Control & $1 \pm 0.24$ & $1 \pm 0.24$ & $1 \pm 0.89$ & $1 \pm 0.23$ & $1 \pm 0.47$ & $1 \pm 0.85$ & $1 \pm 0.33$ \\
\hline $3 w k$ MP & $2.19 \pm 0.47^{*}$ & $3.26 \pm 2.1^{*}$ & $1.87 \pm 1.22$ & $2.82 \pm 0.56^{*}$ & $2.05 \pm 1.79$ & $3.46 \pm 2.34 *$ & $2.18 \pm 1.21 *$ \\
\hline 6wk MP & $4.84 \pm 6.63^{*}$ & $4.03 \pm 6.43^{*}$ & $4.62 \pm 6.41^{*}$ & $3.97 \pm 3.7^{*}$ & $3.82 \pm 3.89^{*}$ & $7.8 \pm 15.56^{*}$ & $3.42 \pm 2.62 *$ \\
\hline 3wk MP/3 wk veh. & $1.01 \pm 0.26$ & $1.39 \pm 0.78$ & $2.12 \pm 1.05$ & $1.28 \pm 0.38$ & $0.88 \pm 0.3$ & $1.14 \pm 0.33$ & $0.68 \pm 0.27$ \\
\hline 14d BDL & $3.88 \pm 1.83 *$ & $25.06 \pm 13.74 *$ & $1.43 \pm 5.09$ & $2.71 \pm 0.44 *$ & $5.78 \pm 8.31 *$ & $2.83 \pm 9.77$ & $8.83 \pm 2.95^{*}$ \\
\hline
\end{tabular}

Values are the mean and SD normalised to control liver. *Significance difference compared to control at $\mathrm{p}<0.05$. For control, 3wk MP 5 and 3 wk MP/3wk vehicle (veh), n=5; for 6wk MP, n=6 and for 14d BDL, $n=8$. 
Figure 1: Chronic MP treatment significantly increased ALP serum activity and liver weight. A. Serum values of ALT, AST and ALP in rats treated with MP for 3 weeks or subjected to BDL for 14 days. B. Liver to total body weight ratio in rats treated with MP for 53 weeks or subject to 14 day BDL. In all cases data are the means and SDs. *significant difference compared to respective control $(\mathrm{p}<0.05)$. Animal numbers - for vehicle, $n=3$; for MP and sham, $n=5$; for BDL, $n=8$.

Figure 2: MP treatment or 14 day BDL caused periportal inflammation. A. H\&E stained sections of rat liver tissue after the indicated treatment. Images are representative of groups and scale bar indicates $100 \mu \mathrm{m}$. Note, control is from the vehicle control group, 10 which had an identical histopathological appearance to sections from sham BDL operated rats, hence only one view is shown and referred to as control. B. Quantified mean number of periportal inflammatory cells. Data are the mean and SD. *significant difference compared to the respective control group, \$significant difference compared to the 3 week MP/3 week vehicle group (both p<0.05). Animal numbers - for control, $n=3$; for $3 w k$ MP, $3 w k$ MP/3 wk vehicle and sham, $n=5$; for $6 w k$ MP, $n=6$; and for BDL $n=8$.

${ }_{15}$ Figure 3: 6 weeks chronic MP treatment or 14 day BDL caused hepatocyte and cholangiocyte proliferation. A. Rat liver sections immunostained for PCNA after the indicated treatment. Images are representative of groups and scale bar indicates $100 \mu \mathrm{m}$. Note, control is from the vehicle control group, which had an identical histopathological appearance to sections from sham BDL operated rats, hence only one view is shown and referred to as control. B. Quantification of periportal PCNA positive hepatocytes and cholangiocytes. Data are mean and SD. *significant difference compared to the respective control group $(\mathrm{p}<0.05)$. Animal numbers - for control, $\mathrm{n}=3$; for 20 sham, $n=4$; for 3 wk MP, 3 wk MP/3 wk vehicle, $n=5$; for $6 w k$ MP, $n=6$; and for BDL $n=8$. A high powered view of a portal tract identifying PCNA positive hepatocytes and cholangiocytes is given in Supplementary Figure 3.

Figure 4. Chronic MP treatment or 14 day BDL caused a significant increase in periportal vimentin staining. A. Rat liver sections immunostained for vimentin after the indicated treatment. Images are representative of groups and scale bar indicates $100 \mu \mathrm{m}$. Note, 25 control is from the vehicle control group, which had an identical histopathological appearance to sections from sham BDL operated rats, hence only one view is shown and referred to as control. B. Quantified vimentin labelling of periportal regions. Values are expressed relative to respective controls. Data are the mean and SD. *significant difference compared to the respective control group and \$significant different compared to the 3 week MP/3 week vehicle group and ${ }^{£}$ significant difference compared to the 3 week MP group (all $\mathrm{p}<0.05$ ). Animal numbers - for control, $\mathrm{n}=3$; for 3 wk MP, 3 wk MP/3 wk vehicle and sham, $n=5$; for $6 w k \mathrm{MP}, \mathrm{n}=6$; and for BDL $\mathrm{n}=8$.

Figure 5. Chronic MP treatment or 14 day BDLcaused significant increases in periportal myofibroblasts. A. Rat liver sections immunostained for $\alpha$-SMA after the indicated treatment. Images are representative of groups and scale bar indicates $100 \mu \mathrm{m}$. Note, control is from the vehicle control group, which had an identical histopathological appearance to sections from sham BDL operated rats, hence only one view is shown and referred to as control. B. Quantification of periportal $\alpha$-SMA positive cells (myofibroblasts). Data are

35 the mean and SD. " significant difference compared to the respective control group, ${ }^{\$}$ significant different compared to the 3 week MP/3 week vehicle group and ${ }^{\mathrm{E}}$ significant difference compared to the 3 week MP group (all $\mathrm{p}<0.05$ ). Animal numbers - for control, $\mathrm{n}=3$; for 3 wk MP, 3wk MP/3 wk vehicle and sham, $n=5$; for $6 \mathrm{wk}$ MP, $n=6$; and for BDL $n=8$. For high powered view of staining, see Supplementary Figure 5 demonstrating cytoplasmic staining.

${ }_{40}$ Figure 6. Chronic MP treatment or 14 day BDL caused significant periportal fibrosis. A. Rat liver sections stained with sirius red after the indicated treatment. Images are representative of groups and scale bar indicates $400 \mu \mathrm{m}$. Note, control is from the vehicle control group, which had an identical histopathological appearance to sections from sham BDL operated rats, hence only one view is shown and referred to as control. B. Quantified sirius red staining of periportal fibrosis, values are expressed relative to respective controls. All individual readings are shown, lower solid lines are the means and the dashed lines are the SD. *Significant difference compared to the 45 respective control group, ${ }^{£}$ significant difference compared to the 3 week MP group $($ all $\mathrm{p}<0.05)$ and ${ }^{\dagger}$ significant difference compared to the 14d BDL group. Animal numbers - for control, n=3; for 3wk MP, 3wk MP/3 wk vehicle and sham, n=5; for 6wk MP, n=6; and for BDL $n=8$.

Figure 7: Chronic MP treatment or 14 day BDL both cause activation of a ductular reaction. A. Rat liver sections stained with ${ }_{50}$ CK-19 after the indicated treatment. Images are representative of groups and scale bar indicates $100 \mu \mathrm{m}$. Note, control is from the vehicle control group, which had an identical histopathological appearance to sections from sham BDL operated rats, hence only one view is shown and referred to as control. B. Quantified CK19 labelling of liver sections, values are expressed relative to respective controls.

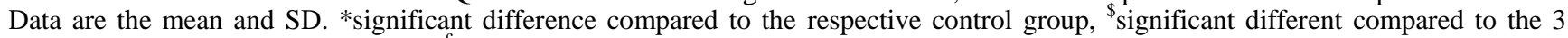
week MP/3 week vehicle group and ${ }^{£}$ significant difference compared to the 3 week MP group (all p<0.05). Animal numbers - for 55 control, $n=3$; for $3 w k$ MP, $3 w k$ MP/3 wk vehicle and sham, $n=5$; for $6 w k$ MP, $n=6$; and for BDL $n=8$.

Supplementary Figure 1: Chronic MP liver injury is associated with neutrophil recruitment to the periportal regions of the liver lobule. A. Rat liver sections stained for esterase. Images are representative of groups and scale bar indicates $100 \mu \mathrm{m}$. B. Quantified esterase staining of periportal regions. Bars are the mean and SD. *significant difference compared to the respective control group and ${ }_{60}$ \$significant different compared to the 3 week MP/3 week vehicle group (all $\mathrm{p}<0.05$ ). Animal numbers - for control, $\mathrm{n}=3$; for $3 \mathrm{wk}$ MP, $3 \mathrm{wk} \mathrm{MP} / 3$ wk vehicle, $n=5$; and for $6 \mathrm{wk}$ MP, $n=6$.

Supplementary Figure 2: Chronic MP treatment did not alter spleen or lung pathology. A. H\&E stained sections of rat lung or spleen tissue in control or 6 week MP treated rats. Images are representative of groups and scale bar indicates $100 \mu \mathrm{m}$.

65

Supplementary Figure 3: PCNA immunostain in a MP-treated rat liver section. High magnification of liver periportal region immunostained for PCNA indicating typical PCNA positive hepatocytes (full arrows) and typical positive cholangiocytes (dotted arrows). 
Supplementary Figure 4: Chronic MP and BDL caused significant hepatic fibrosis. A. Quantification of sirius red staining. B. Liver hydroxyproline content following the indicated treatments. Data are expressed relative to respective controls and are the mean + SD. *Significant difference compared to the control group. Animal numbers - for control, $n=3$; for $3 w k$ MP and $3 w k ~ M P / 3$ wk vehicle, $n=5$; 6wk MP, $n=6$; sham, $n=8$ and BDL, $n=12$.

5

Supplementary Figure 5: $\alpha$-SMA immunostain in a MP-treated rat liver section. High magnification of liver periportal region immunostained for $\alpha$-SMA illustrating cytoplasmic staining myofibroblast cells. a, vessel region, also showing several bile ducts negative for $\alpha$-SMA; b parenchymal region. 

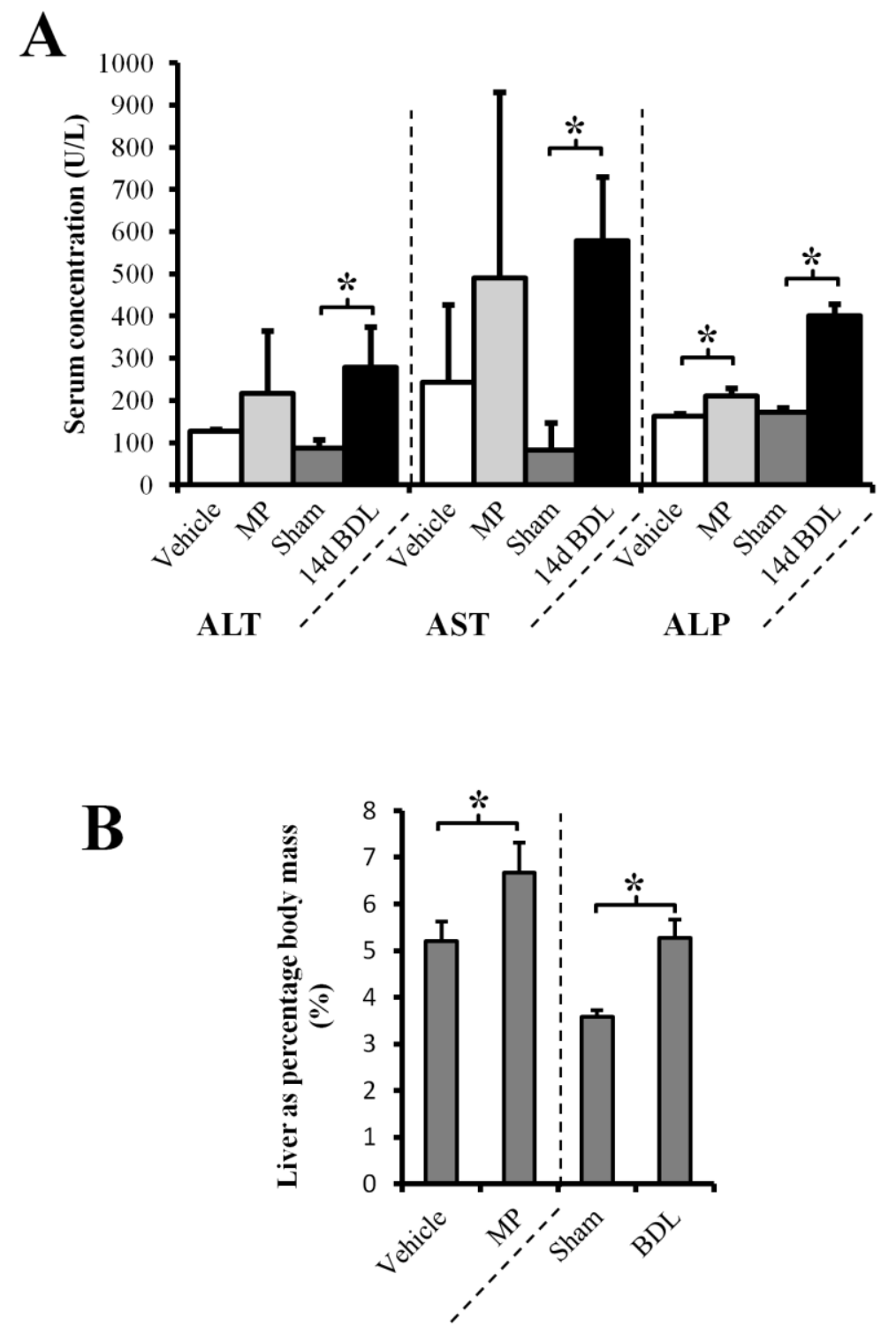

Fig 1 

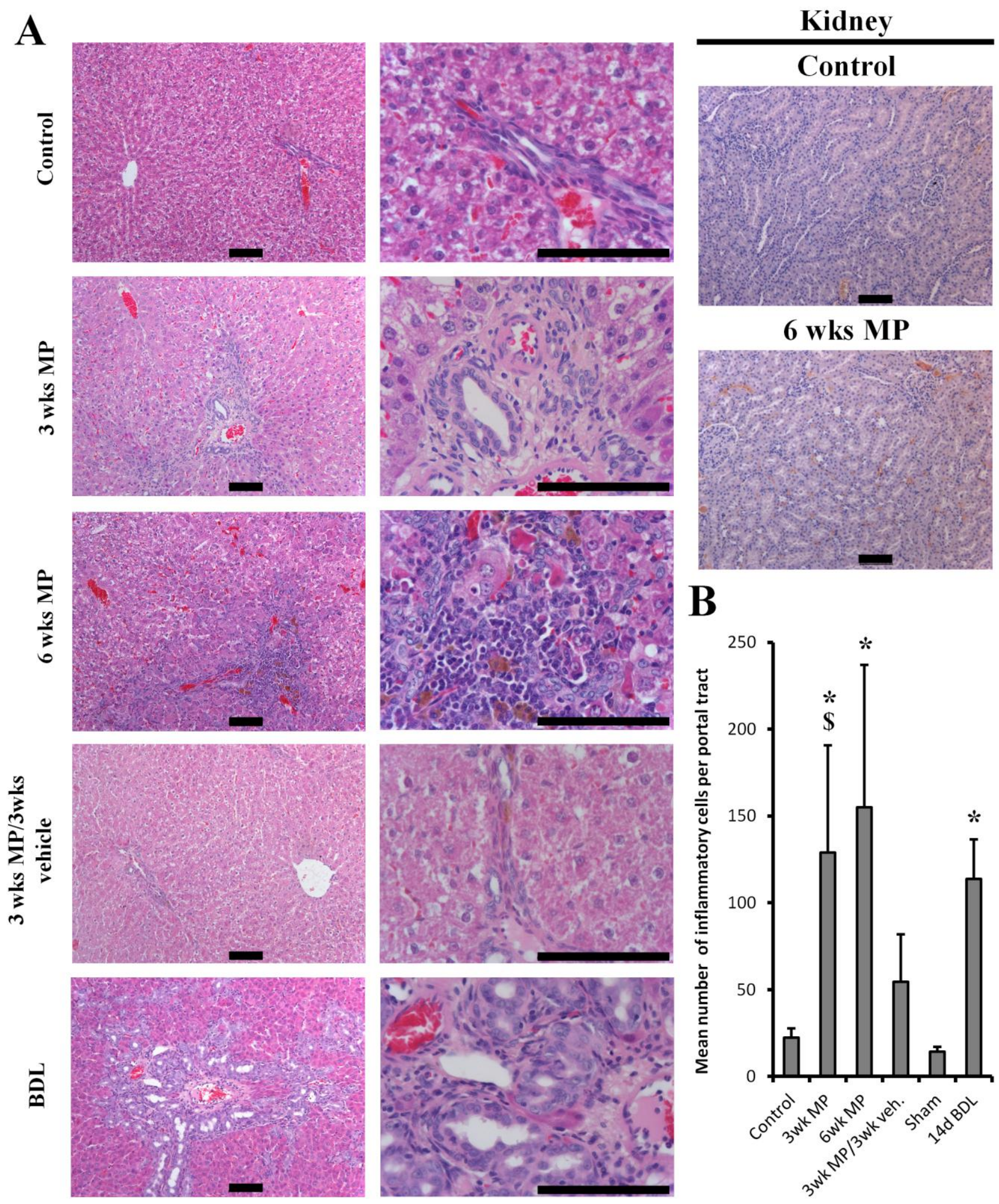

Fig 2 

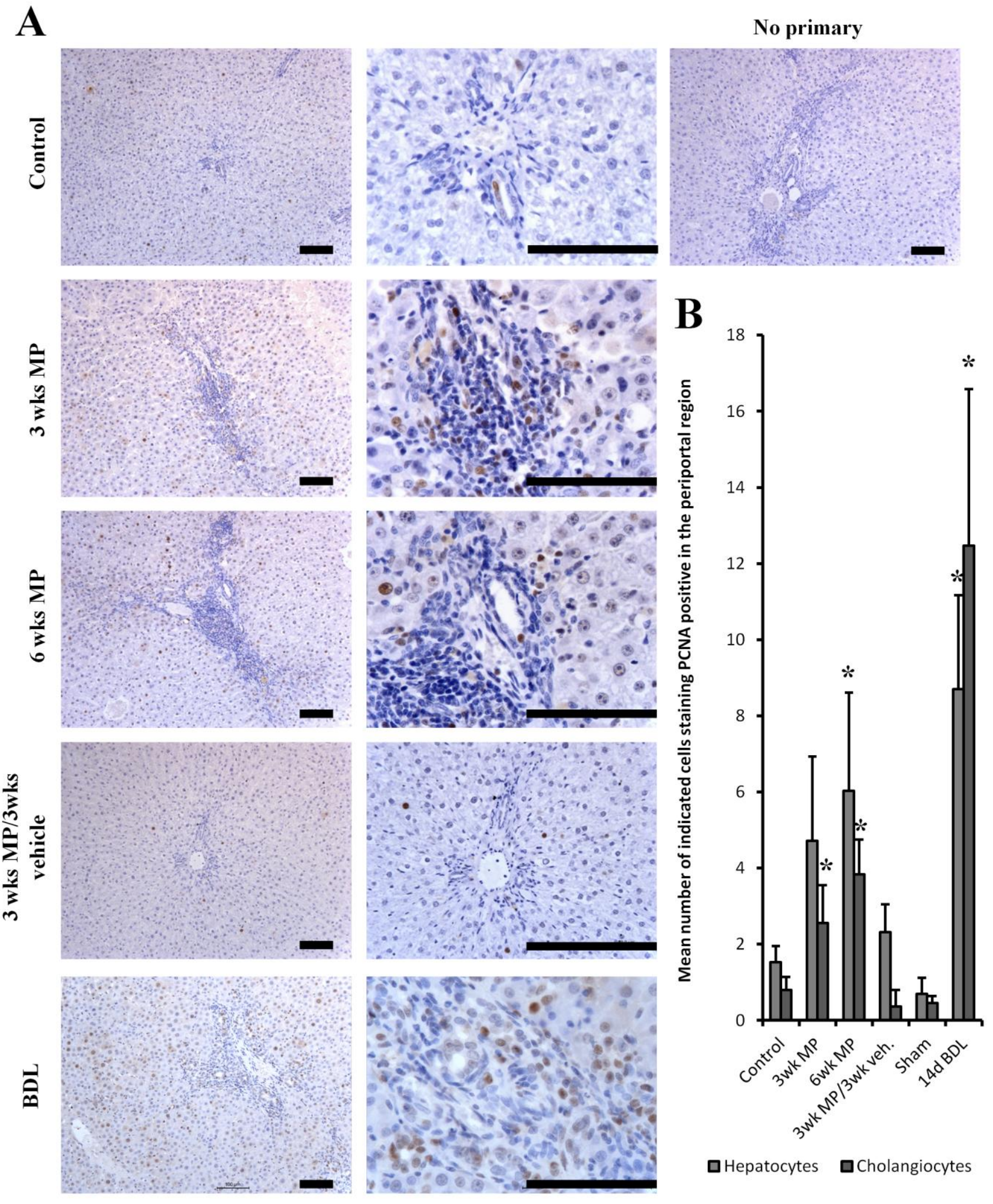

口Hepatocytes $\mathbf{a}$ Cholangiocytes

Fig 3 
A

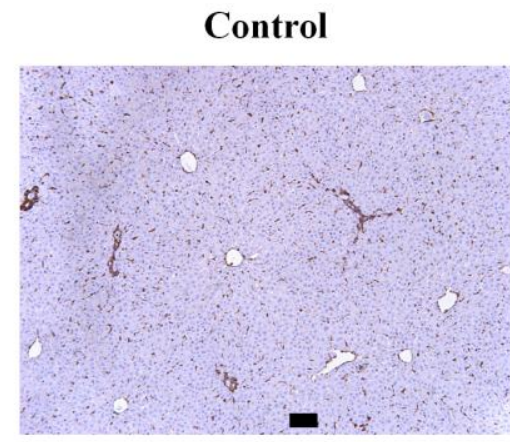

3 wks MP

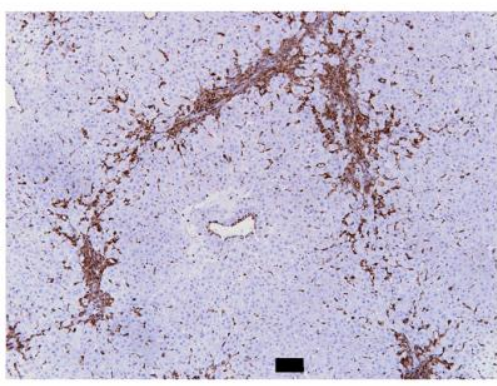

BDL

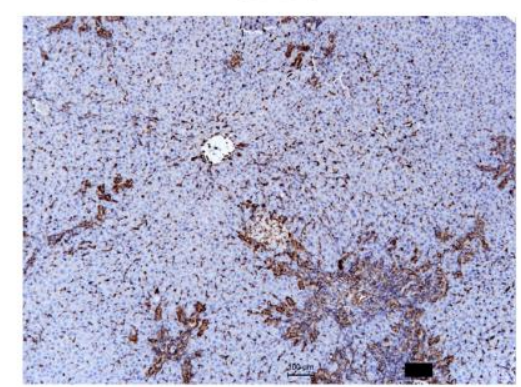

6 wks MP

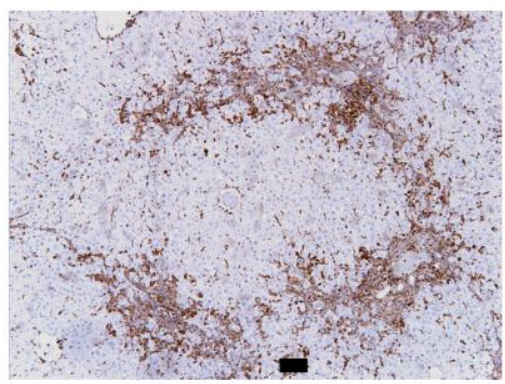

3 wks MP/3wks vehicle

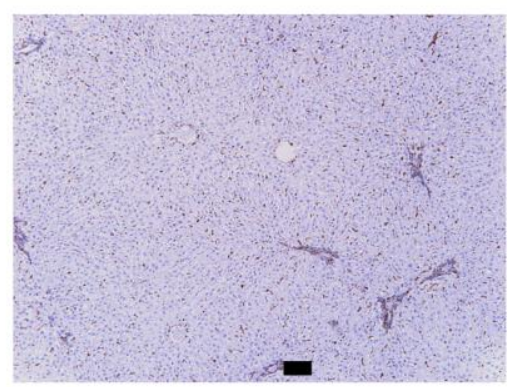

No primary

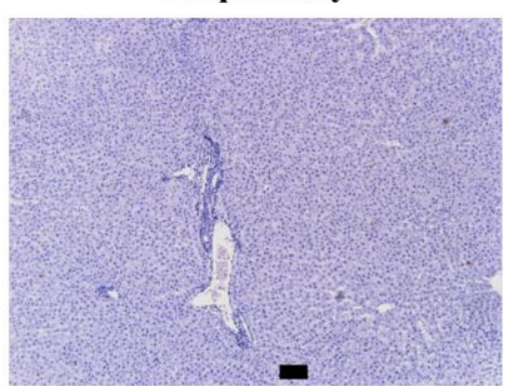

B

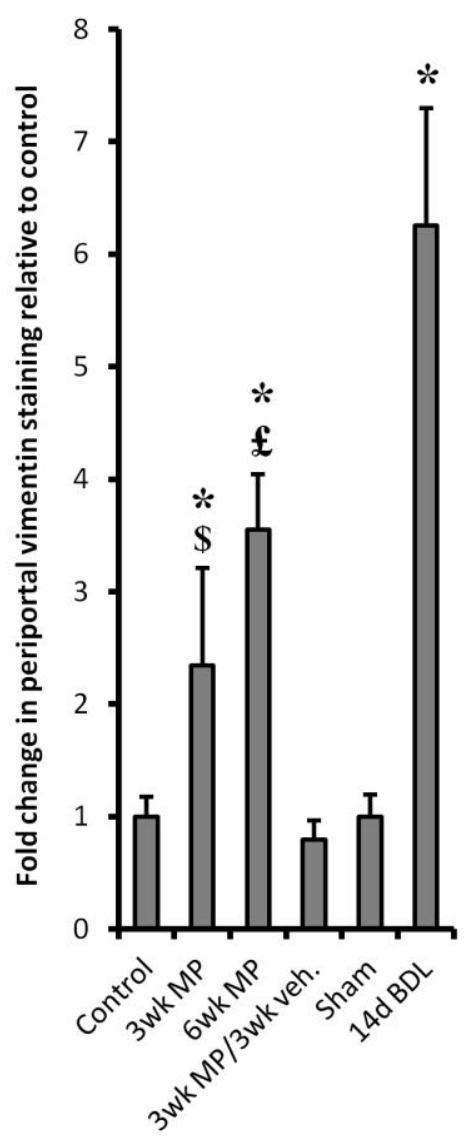

Fig 4 

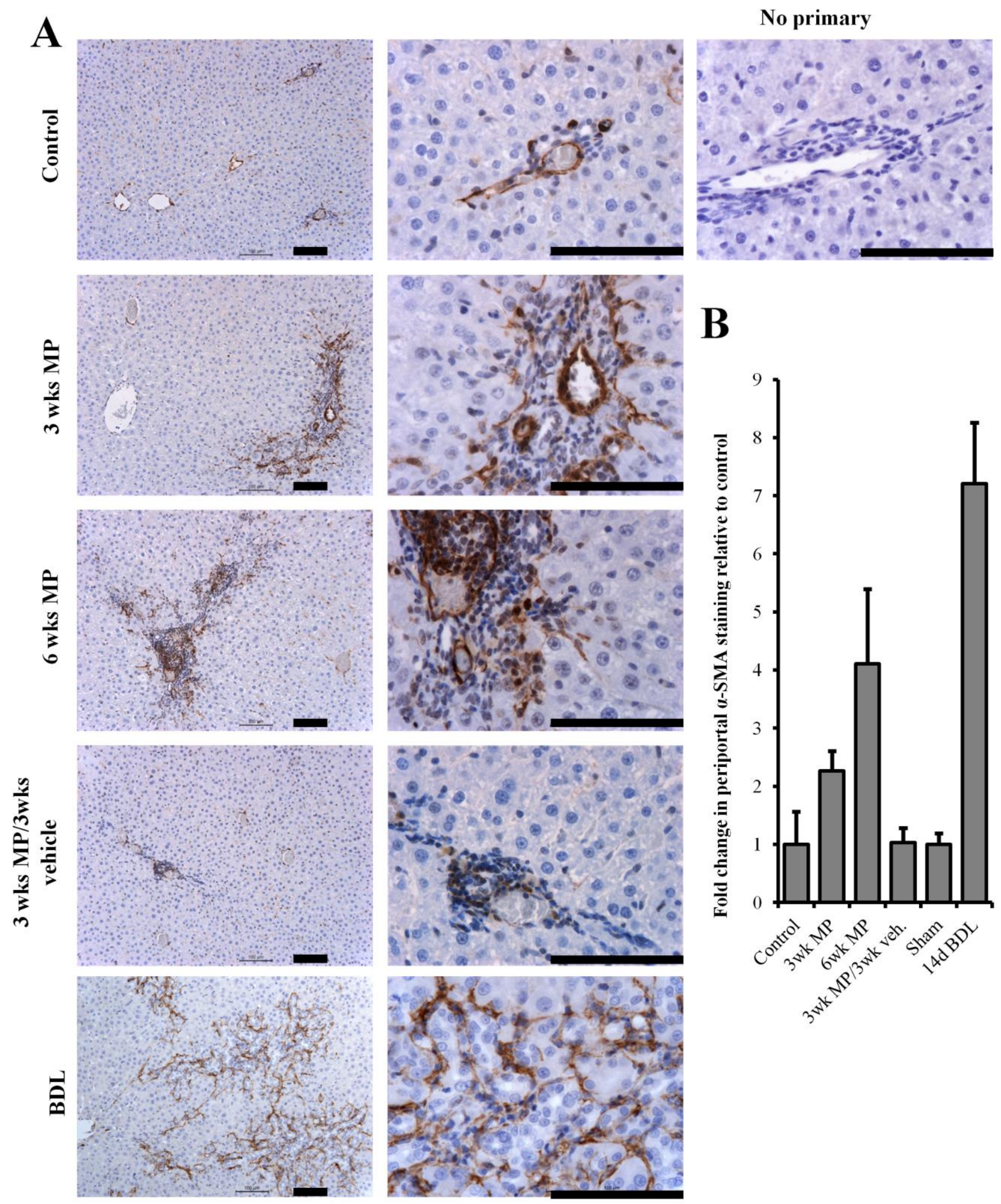

\section{B}

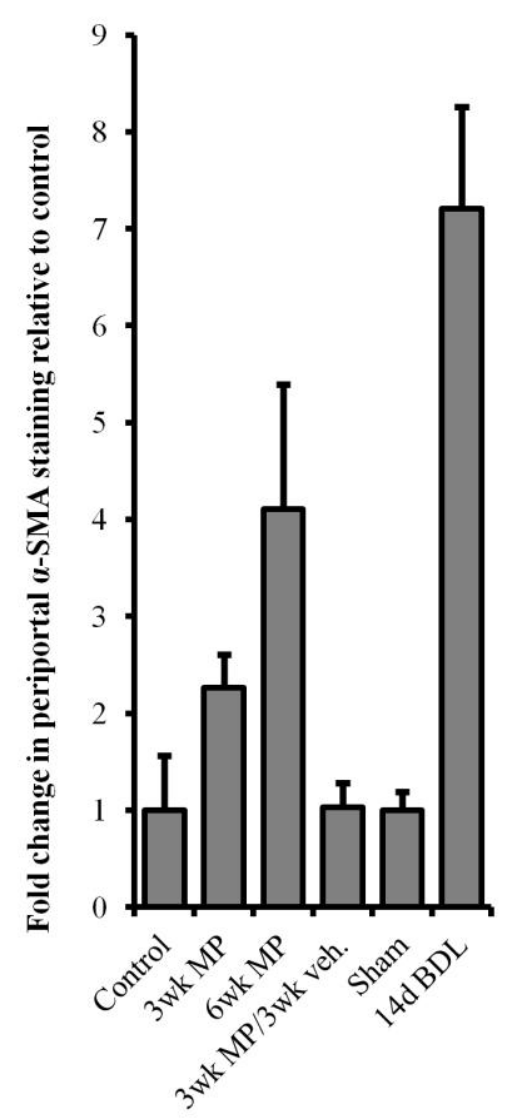

Fig 5 


\section{Control}

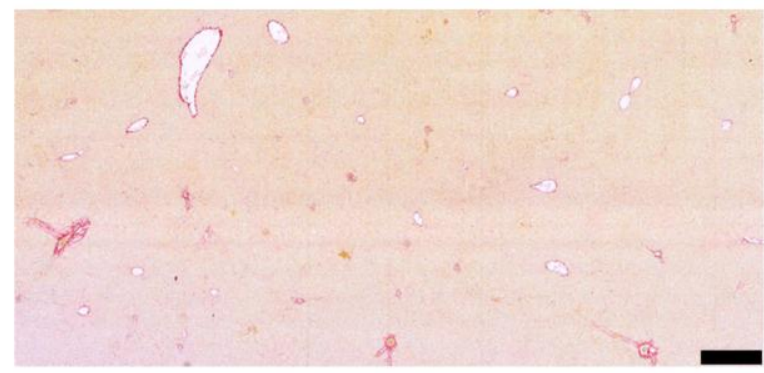

3 wks MP

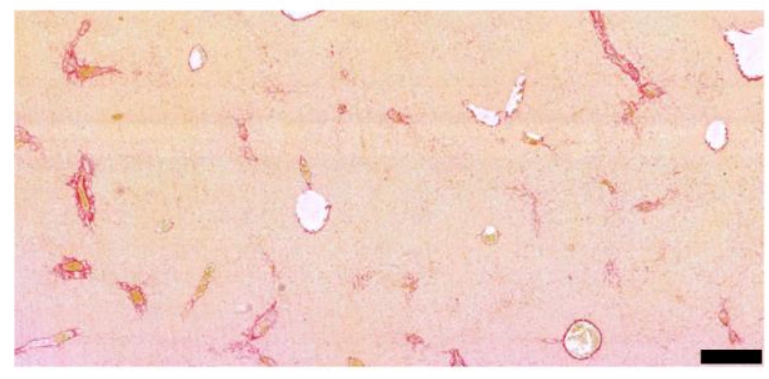

6 wks MP

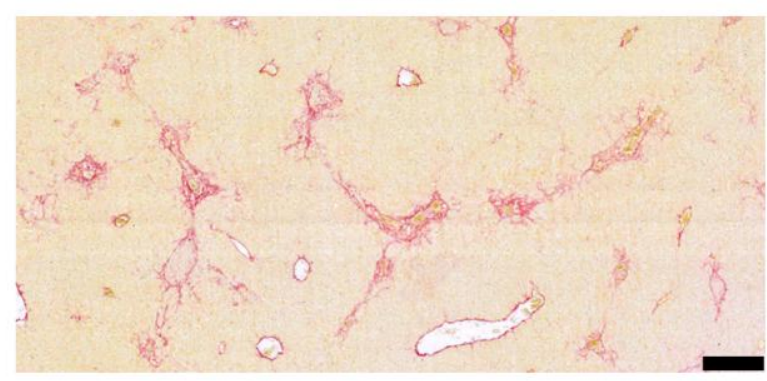

14d BDL

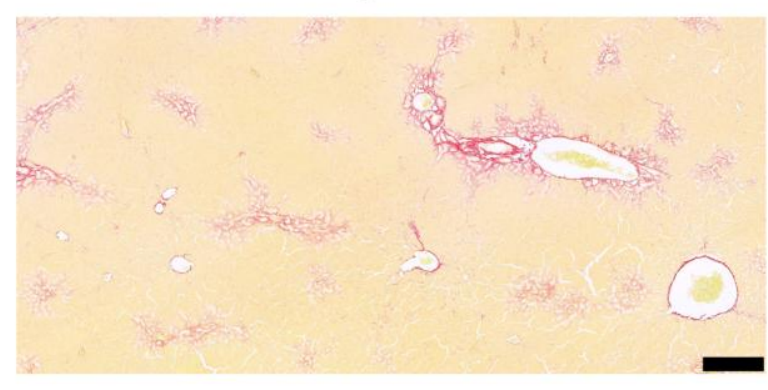

3 wks MP/3wks vehicle
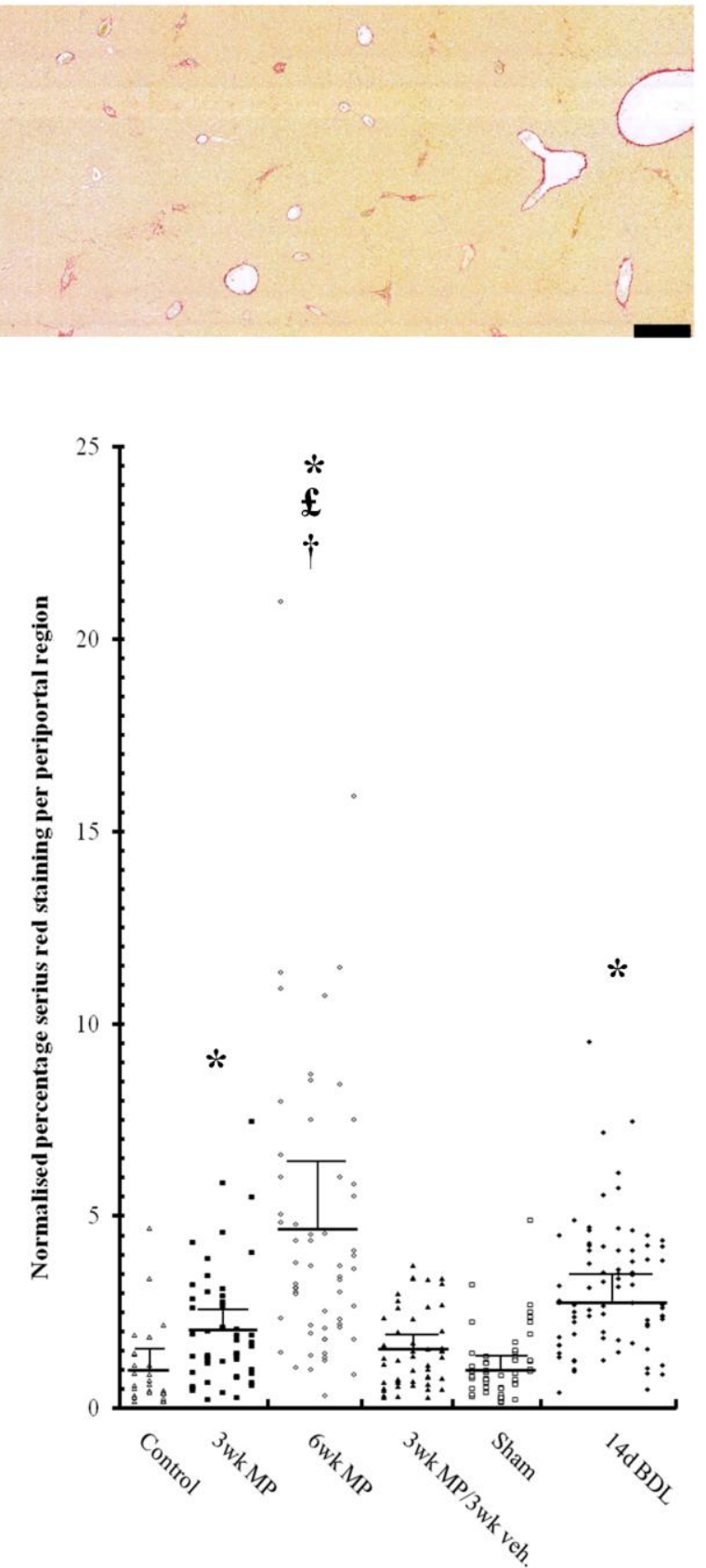

Fig 6 


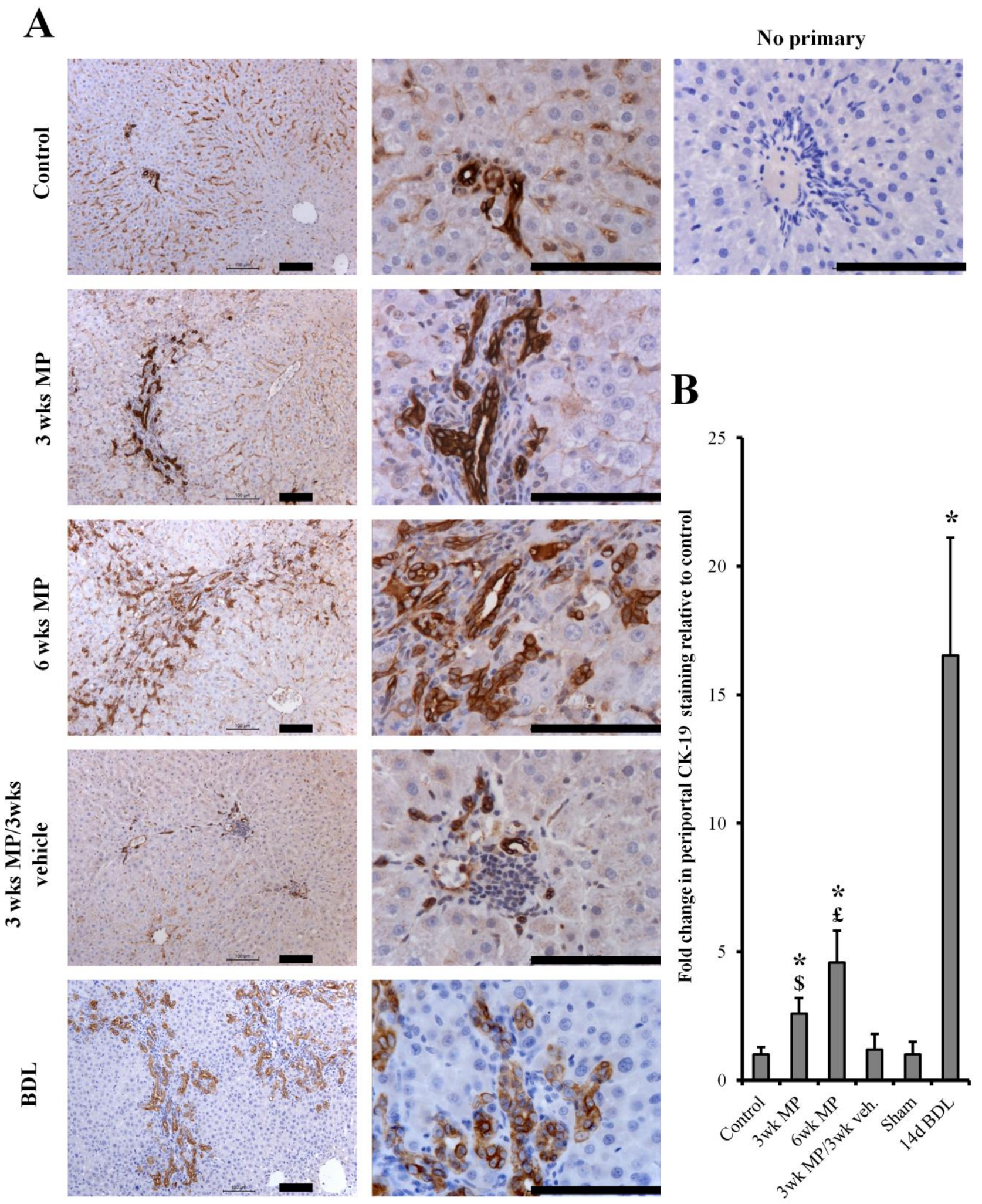

Fig 7 


\section{Supplementary Data}
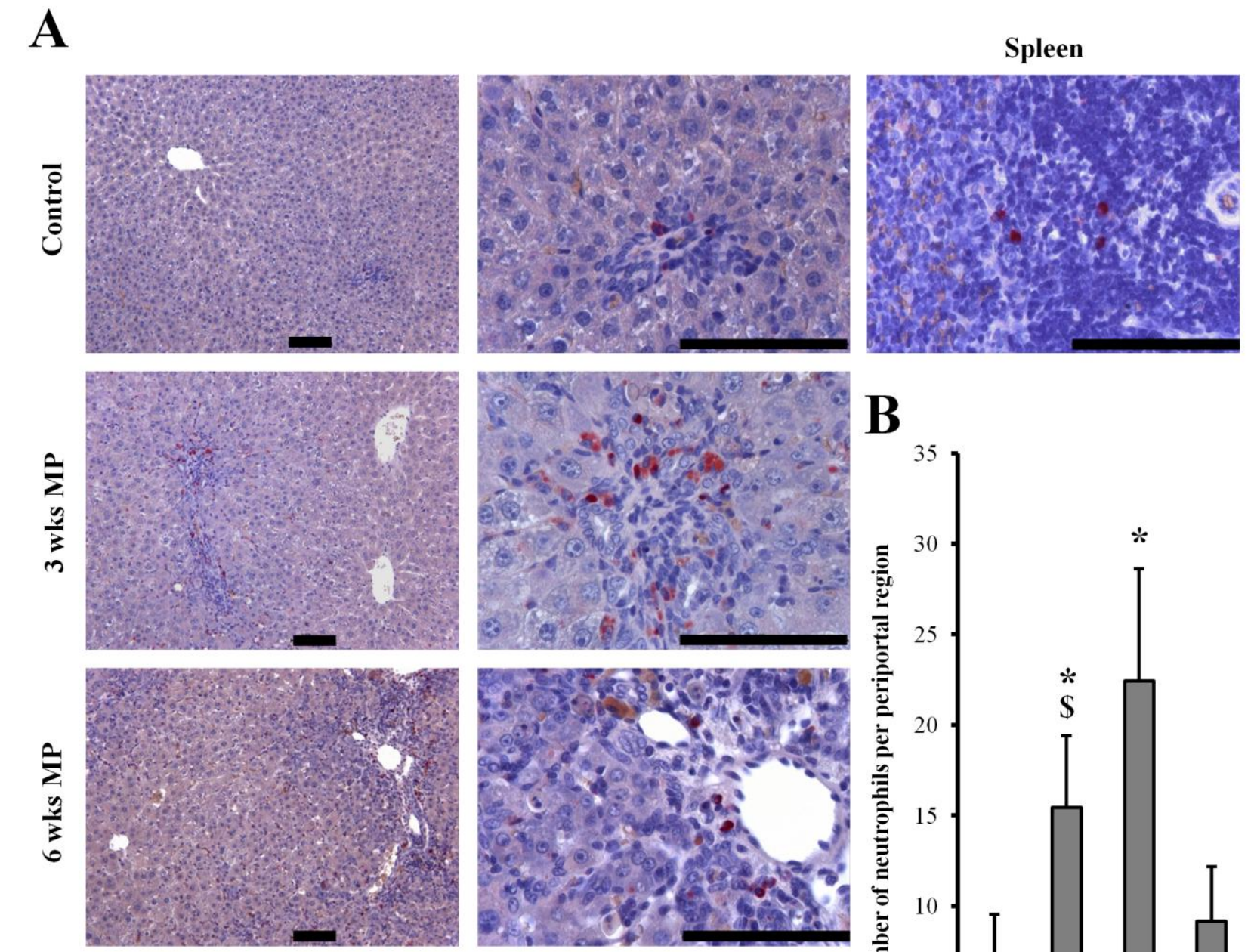

\section{B}

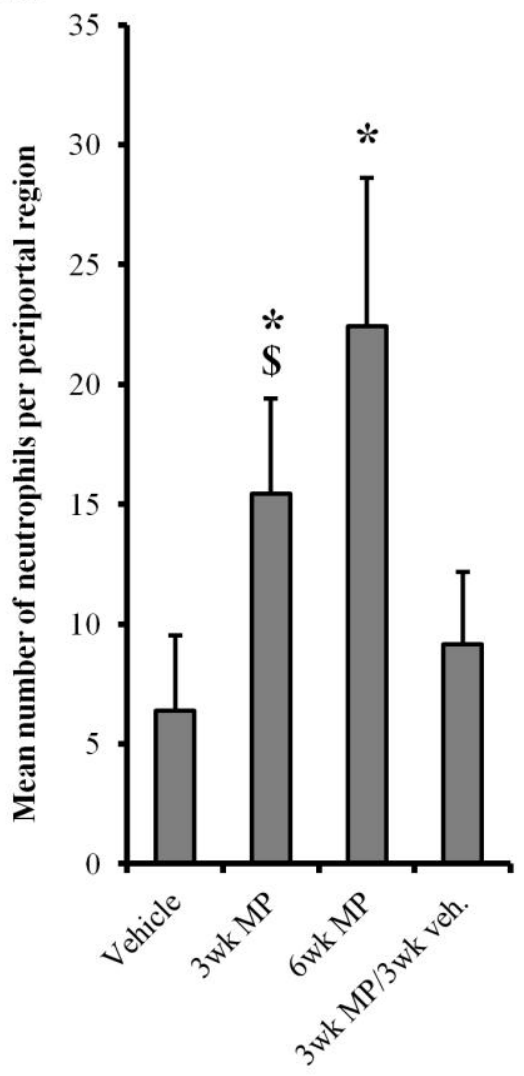

Supplementary Figure 1 

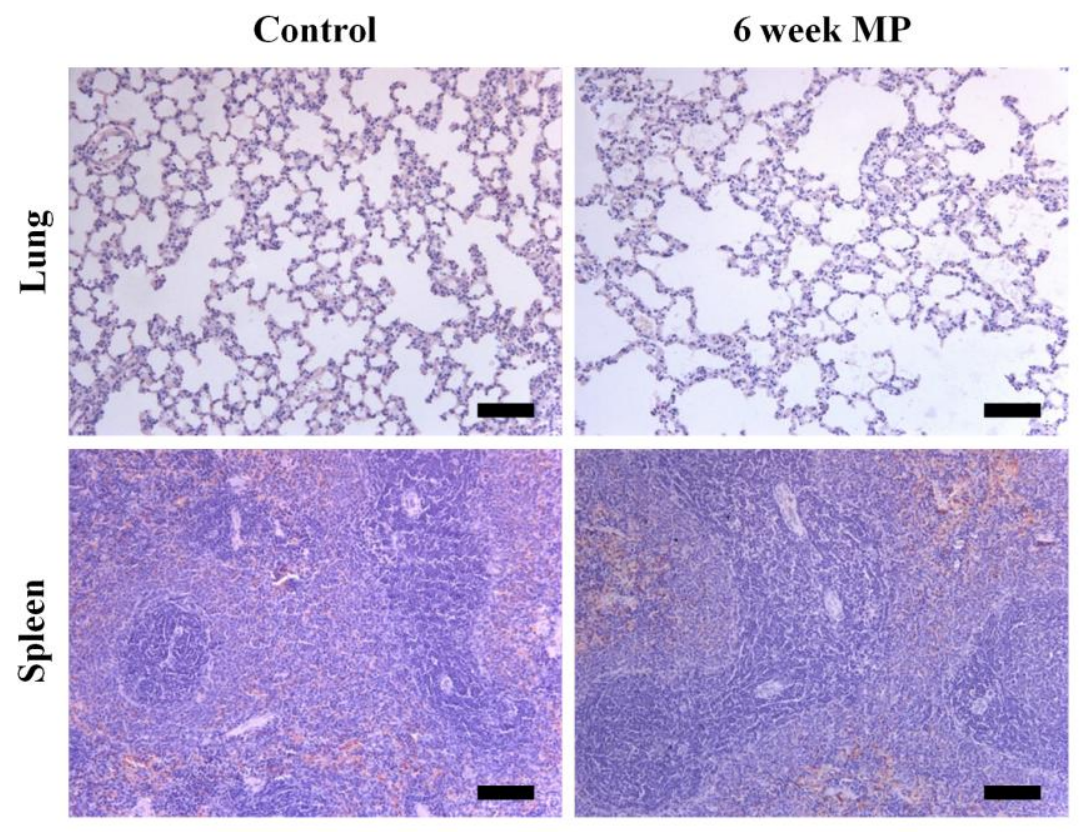

\section{Supplementary Figure 2}



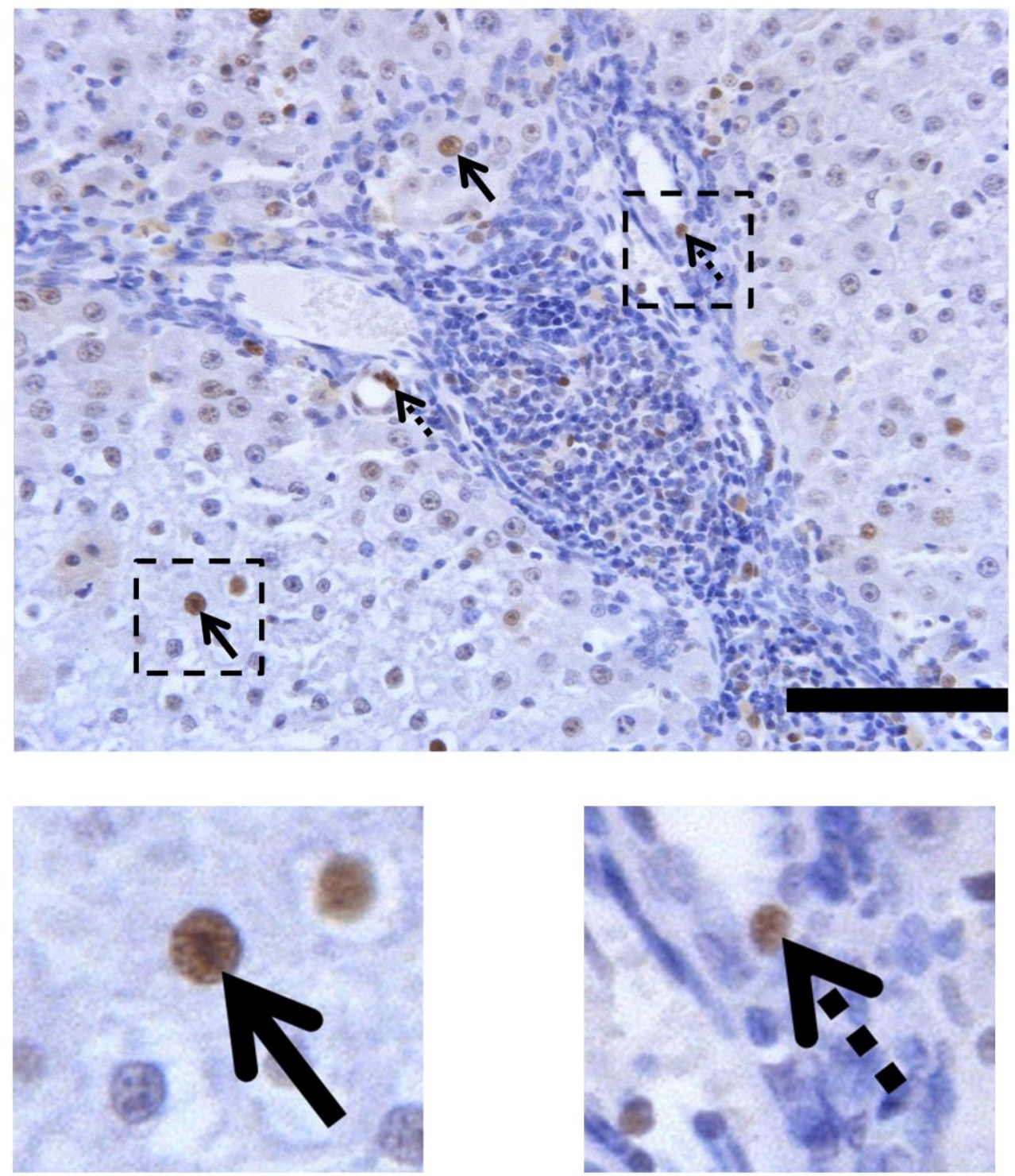

\section{Supplementary Figure 3}



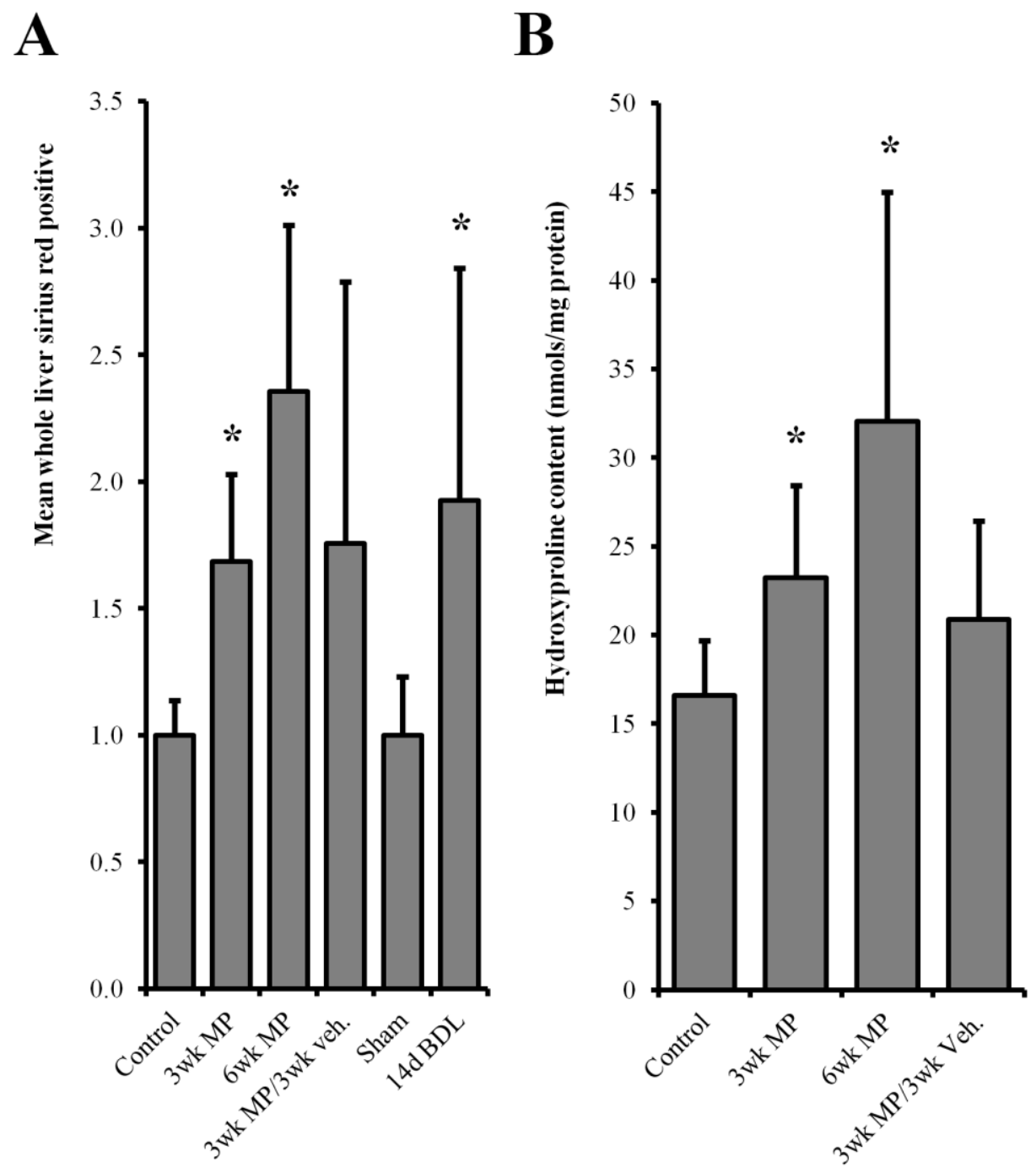

Supplementary Figure 4 

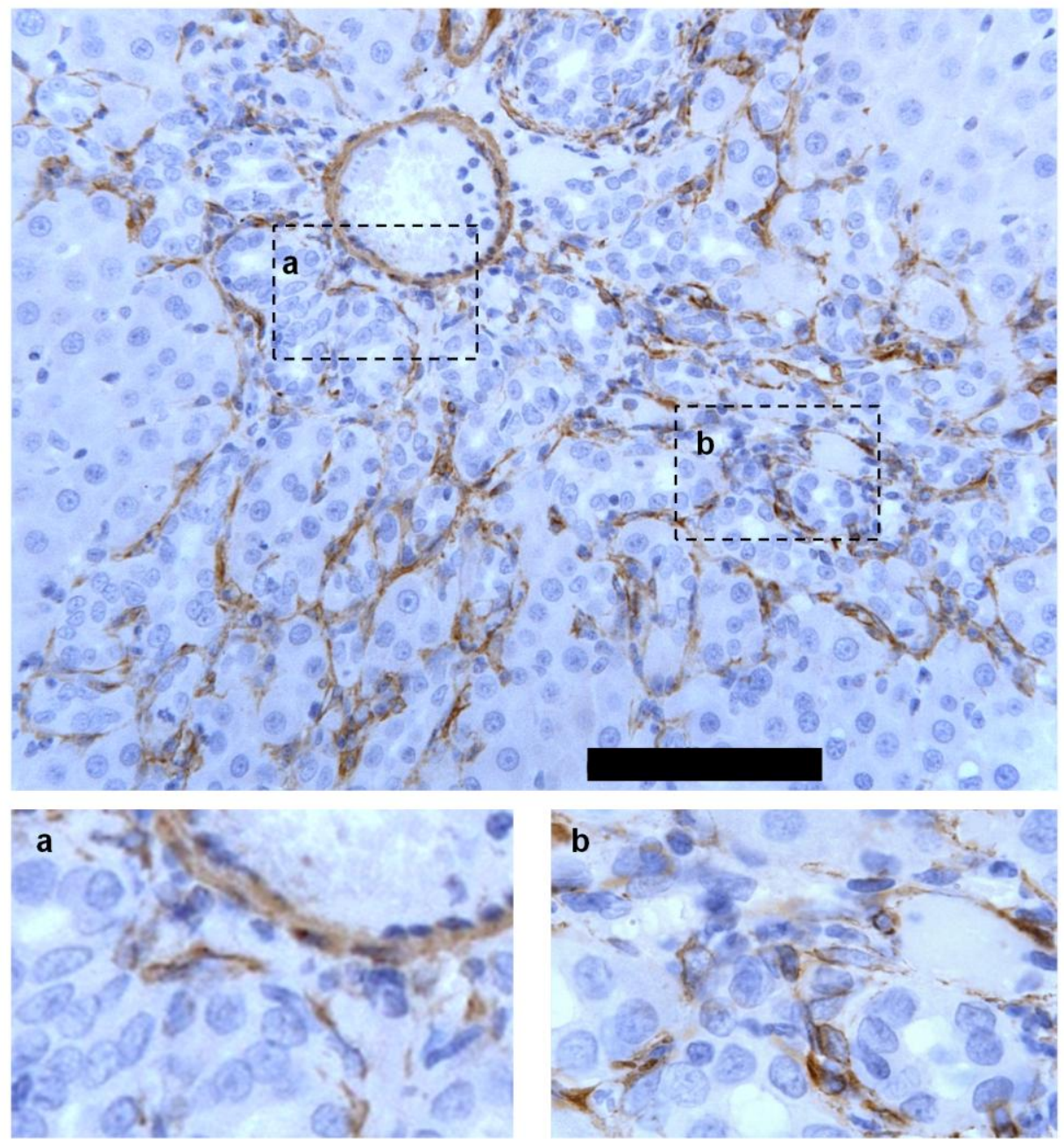

\section{Supplementary Figure 5}

